\title{
Tuberculose em População Indígena Autodeclarada no Estado do Paraná
}

\section{Tuberculosis in Self-declares Indigenous People in the State of Parana}

\author{
Sidimara Sakser Zorzi ${ }^{1}$ \\ Regiane Bezerra Campos ${ }^{2}$ \\ Samia Regina de Quadros ${ }^{3}$ \\ Adriana Zilly ${ }^{4}$ \\ Pedro Fredemir Palha ${ }^{5}$ \\ Betina Mendez Alcântara Gabardo ${ }^{6}$ \\ Reinaldo Antonio Silva-Sobrinho ${ }^{7}$
}

\section{RESUMO}

Objetivo: Descrever o perfil epidemiológico e o percentual de cura da tuberculose (TB) na população indígena no Estado do Paraná. Metodologia: Trata-se de um estudo epidemiológico retrospectivo realizado a partir de 36.889 casos de tuberculose obtidos por meio do Sistema de Informação de Agravos de Notificação do Estado do Paraná, no período de 2001 a 2012. Foram selecionadas variáveis clínicas contemplando dados da população indígena notificada com tuberculose no Estado do Paraná, forma clínica da tuberculose, situação de encerramento e variáveis sociodemográficas (sexo, idade, escolaridade e zona de domicílio). Utilizou-se análise exploratória das variáveis por meio da distribuição de frequência absoluta e relativas. Resultados: Considerando a população total do estudo, 174 eram indígenas com predomínio de tuberculose na forma pulmonar (85\%), apresentando coeficiente de incidência elevado principalmente no sexo masculino $(58,6 \%)$, em residentes na zona rural $(67,2 \%)$, sendo que, $72,4 \%$ obtiveram a cura. A faixa etária com idade entre $31-40$ anos $(20,1 \%)$ apresentou o maior número de casos notificados. Conclusão: A população que se declarou indígena apresentou características sociodemográficas similares à população geral com tuberculose, entretanto, evidenciou-se vulnerabilidade na população declarada indígena para incidência e cura da doença.

DESCRITORES: Tuberculose; População indígena; Epidemiologia.

\section{ABSTRACT}

Objective: To describe the epidemiological profile and cure rate of tuberculosis (TB) in the indigenous population in the state of Paraná. Methodology: This is a retrospective epidemiological study based on 36.889 cases of tuberculosis obtained through the Paraná Notification of Injury Information System from 2001 to 2012. Clinical variables were selected contemplating data from the indigenous population reported with tuberculosis in the State of Paraná, clinical form of tuberculosis, closure and sociodemographic variables (sex, age, schooling and domicile area. An exploratory analysis of the data was used through the absolute and relative frequency distribution. Results: Considering the total population of the study, 174 were indigenous with a predominance of tuberculosis in the pulmonary form $(85 \%)$, with a high incidence rate, mainly in males $(58,6 \%)$, in rural residents $(67,2 \%)$, being that $72,4 \%$ obtained a cure. The age group between $31-40$ years $(20,1 \%)$ had the highest number of reported cases. Conclusion: The population that declared themselves indigenous had sociodemographic characteristics similar to the general population with tuberculosis. However, it showed a vulnerability in the population declared indigenous for incidence and cure of the disease.

DESCRIPTORS: Tuberculosis. Indigenous Population. Epidemiology.

1- Enfermeira. Foz do Iguaçu, PR, Brasil.

2- Doutoranda do Programa de Pós-Graduação Enfermagem em Saúde Pública da Escola de Enfermagem de Ribeirão Preto da Universidade de São Paulo - EERP/USP. Ribeirão Preto, SP, Brasil.

3- Enfermeira. Foz do Iguaçu, PR, Brasil.

4- Professora Associada da Universidade Estadual do Oeste do Paraná - Unioeste. Foz do Iguaçu, PR, Brasil.

5- Professor Titular da Universidade de São Paulo. Escola de Enfermagem de Ribeirão Preto, EERP/USP. Ribeirão Preto. SP. Brasil.

6- Professora Adjunta da Faculdade Pequeno Príncipe, PR, Brasil.

7- Professor Adjunto da Universidade Estadual do Oeste do Paraná - Unioeste. Foz do Iguaçu, PR, Brasil. 
A Tuberculose (TB) é conhecida em todo o mundo por se tratar de uma doença infectocontagiosa, causada pelo Mycobacterium tuberculosis, também chamado Bacilo de Koch¹, a doença se dissemina entre grupos que apresentam maior vulnerabilidade socioeconômica. A falta de saneamento básico, urbanização desregulada com grandes concentrações populacionais desnutrição, o alcoolismo, HIVIAIDS, diabetes e condições de moradia desfavoráveis são fatores que colaboram para a perpetuação da doença na sociedade ${ }^{2}$.

De acordo com a Organização Mundial da Saúde (OMS) a TB se mantém com taxas elevadas nos países subdesenvolvidos, os quais apresentam um elevado índice de pobreza ${ }^{3,4}$. Apesar de ações continuas contra a doença, ela vem permanecendo como a $3^{\circ}$ causa de morte por doenças infecciosas no Brasil ${ }^{5}$.

Aproximadamente, 70 mil novos casos (incidência 36,4/100 mil habitantes) ${ }^{6}$ são diagnosticados anualmente, causando a morte de 4,5 mil pessoas ${ }^{4}$. O país ocupa a $20^{a}$ posição na classificação de carga da doença e a 19a quanto à coinfecção TB/HIV entre os 48 países que mais apresentam casos de TB, os quais totalizam $87 \%$ do número globalmente ${ }^{4}$.

O Brasil possui uma população autodeclarada indígena de 817.963 indivíduos $(0,42 \%$ da população residente do país). Deste total, 53,6\% residem em terras indígenas e $46,4 \%$ residem fora delas. A população indígena do país possui 305 etnias e 274 idiomas, vivendo em $80,5 \%$ dos municípios brasileiros ${ }^{7}$.

Segundo o censo de 2010, no Estado do Paraná residem $28.899^{8}$ índios que pertencem a três etnias: Guarani, Kaingang e Xetá, sendo que a grande maioria vive em terras indígenas demarcadas pelo Governo Federal. O estado possui 18 territórios indígenas regularizados, espalhados ao longo do seu território ${ }^{9}$.

O coeficiente de incidência $(\mathrm{Cl})$ de TB na população indígena no ano de 2010 foi de 93,5 casos /100 mil habitantes, sendo este valor 2,6 vezes maior que na população geral. No ano de 2012 foram diagnosticados 782 novos casos TB entre os indígenas, esse valor representa $1,1 \%$ de todos os casos notificados no país².
Sendo assim, o Programa Nacional de Controle da Tuberculose (PNCT) define o índio como uma população prioritária quando se trata de $\mathrm{TB}$, pois compõe um grupo com vulnerabilidade, tanto econômica quanto social ${ }^{10}$. Apesar do tratamento da TB ser gratuito no Brasil, ela é ainda uma das grandes causas de morbidade e mortalidade, colaborando para o decrescimento da população geral e indígena no país ${ }^{11}$.

$\mathrm{O}$ alto risco de adoecimento por TB entre os indígenas, expressivamente maior que na população geral, o elevado coeficiente de incidência e mortalidade, determinantes culturais envolvidos e a escassez de informações científicas sobre o perfil epidemiológico da população autodeclarada indígena, justificam a realização deste estudo, que teve por objetivo descrever o perfil epidemiológico e o percentual de cura da tuberculose (TB) na população indígena no Estado do Paraná.

\section{METODOLOGIA}

Tratou-se de estudo epidemiológico descritivo, retrospectivo, com abordagem quantitativa. Os dados dos casos de tuberculose no Estado do Paraná foram obtidos por meio do banco de dados do Sistema de Informação de Agravos de Notificação do Paraná (SINAN-PR), no período de 2001 a 2012.

O estudo envolve uma população de 36.889 casos de TB notificadas entre os anos de 2001 a 2012, no Estado do Paraná. Para atender o objetivo da pesquisa foram selecionados todos os casos de TB notificados entre indígenas, conformando 174 casos.

Os dados sobre o quantitativo populacional autodeclarado, da raça/cor indígena residentes no Estado do Paraná foram obtidos no escritório do IBGE/PR.

Para atender o estudo, selecionaram-se as variáveis sociodemográficas (sexo, idade, escolaridade e zona de domicílio) e as variáveis clínicas (forma clínica, tratamento diretamente observado e situação de encerramento). Para o cálculo do $\mathrm{Cl}$ da TB aplicou-se a seguinte equação matemática: número de casos novos de TB (em indígenas de 
todas as formas clínicas) confirmados e residentes no Paraná segundo período do estudo, dividido pela população indígena autodeclarada estimada para os anos estudados (conforme IBGE, 2010), multiplicado por cem mil; para acura o cálculo foi realizado em percentuais.

Os resultados foram analisados tendo como referência as recomendações do manual do Ministério da Saúde ${ }^{12}$.

Esta pesquisa é um recorte do projeto PPSUS - "Epidemiologia de Doença Negligenciada: Comportamento Espacial e Temporal da Incidência da Tuberculose no Estado do Paraná", aprovado pelo Comitê de Ética e Pesquisa envolvendo seres humanos, obedecendo à resolução CNS $n^{\circ}$. 446/2012, parecer $n^{\circ}$. 102/2013.

\section{RESULTADOS}

Foram encontrados dados de 174 casos de TB na população que se declarou indígena entre os anos de 2001 a 2012 no estado do Paraná.

A maior incidência foi encontrada entre pessoas do sexo masculino, $58,6 \%$ do total dos doentes. A faixa etária com idade entre 31-40 anos $(20,1 \%)$ apresentou o maior número de casos notificados. Destaca-se ainda que os indivíduos com escolaridade entre $1^{\mathrm{a}}$. a $4^{\mathrm{a}}$. série do ensino fundamental incompleto $(20,6 \%)$ apresentaram maior índice da doença. $O$ estudo analisou o número de casos da TB em relação à localidade da residência, sendo encontrada a maior incidência da doença em indígenas residentes na zona rural $(67,2 \%)$ do estado (Tabela 1$)$.

A TB pulmonar foi à forma clínica mais comum, presente em $86,2 \%$ dos casos diagnosticados. Dentre os indivíduos doentes $54,0 \%$ realizavam o tratamento diretamente observado (TDO). Dos 174 casos de TB notificados entre índios, $72,4 \%$ obtiveram situação de encerramento satisfatório, obtendo a cura da doença (Tabela 2).

Os valores calculados de $\mathrm{Cl}$ apresentaram uma variabilidade elevada ao longo dos anos, podendo ressaltar os anos de $2002 \mathrm{com} \mathrm{Cl}$ de 320 casos/100 mil habitantes e o ano de $2010 \mathrm{com}$ 111,5 casos/100 mil habitantes. Os menores $\mathrm{Cl}$ para a população indígena, ainda que elevados, foram verificados nos anos de 2001 e 2009 com $\mathrm{Cl}$ de 34,8 e 33,3 casos/100 mil habitantes, respectivamente (Tabela 3 ).

Quando comparado o $\mathrm{Cl}$ e a porcentagem de cura, observou-se que em 2002 o Cl atingiu 320 casos /100 mil habitantes, e o percentual de cura foi de $62,5 \%$. Destaca-se que nos anos em que - $\mathrm{Cl}$ apresentou valores menores a porcentagem de cura apresentou-se de forma satisfatória. No entanto, nos anos em que o $\mathrm{Cl}$ se manteve elevado o percentual de cura não alcançou o parâmetro desejado, salvo em 2001 e 2004 no qual o percentual de cura foi maior que $85 \%$.

Observou-se que independente do $\mathrm{Cl}$, o comportamento da curva da cura não apresentou tendência para se manter acima de $85 \%$ período (gráfico 1)

Gráfico 1. Coeficiente de incidência versus percentual de cura entre indígenas no estado do Paraná no período 2001 a 2012.

\section{DISCUSSÃO}

A análise da ocorrência de casos de TB na população indígena segundo as condições sociodemográficas, no Estado do Paraná revela semelhança com o padrão encontrado na população geral, confirmando a vulnerabilidade social e econômica dos grupos onde há maior incidência da doença $a^{13,14}$.

As populações socioeconomicamente afetadas são as mais vulneráveis à TB, sendo que os povos indígenas fazem parte do grupo suscetível ${ }^{15}$.

Os indígenas se destacam na região amazônica com incidências de TB que ultrapassam 1000/100 000 habitantes $^{16}$, à medida que as médias nacionais se estabelecem na faixa de $40 / 100000$ habitantes $^{17}$. Ao analisar a ocorrência da doença com base na idade verificou-se mesmo padrão da população geral. Contudo, uma população mais jovem quando comparada a um estudo realizado no Amazonas, onde a maior concentração de TB em indígenas estava entre indivíduos acima da faixa de 50 anos $^{18}$. 
Tabela 1. Distribuição das variáveis epidemiológicas e sociodemográficas dos indígenas do Estado do Paraná nos anos de 2001 a 2012.

\begin{tabular}{|c|c|c|c|c|c|c|c|c|c|c|c|c|c|c|}
\hline \multirow{2}{*}{ Variáveis } & \multicolumn{13}{|c|}{$n^{0}$} & \multirow[b]{2}{*}{$\%$} \\
\hline & 2001 & 2002 & 2003 & 2004 & 2005 & 2006 & 2007 & 2008 & 2009 & 2010 & 2011 & 2012 & Total & \\
\hline \multicolumn{15}{|l|}{ Sexo } \\
\hline Feminino & 3 & 15 & 4 & 3 & 6 & 4 & 7 & 4 & 2 & 16 & 5 & 3 & 72 & 41,3 \\
\hline Masculino & 5 & 16 & 10 & 3 & 6 & 10 & 8 & 10 & 9 & 13 & 4 & 8 & 102 & 58,6 \\
\hline Total & 8 & 31 & 14 & 6 & 12 & 14 & 15 & 14 & 11 & 29 & 9 & 11 & 174 & 100 \\
\hline \multicolumn{15}{|l|}{ Idade (anos) } \\
\hline $0-10$ & 2 & - & 2 & 3 & 2 & 3 & 4 & 3 & 3 & 1 & 1 & - & 24 & 13,8 \\
\hline $11-20$ & 2 & 3 & 2 & - & 1 & 2 & 1 & - & - & 5 & 3 & 2 & 21 & 12 \\
\hline $21-30$ & 2 & 5 & 1 & 1 & 3 & 1 & 3 & 4 & 5 & 6 & 2 & - & 33 & 19 \\
\hline $31-40$ & 2 & 5 & 4 & 2 & 2 & 6 & 1 & 2 & 1 & 3 & 2 & 5 & 35 & 20,1 \\
\hline $41-50$ & - & 4 & 3 & - & 1 & - & 2 & 2 & - & 6 & - & 3 & 21 & 12 \\
\hline $51-60$ & - & 4 & - & - & 2 & 2 & 3 & 2 & 1 & 3 & 1 & 1 & 19 & 11 \\
\hline $61-70$ & - & 6 & 1 & - & - & - & 1 & 1 & - & 2 & - & - & 11 & 6,32 \\
\hline $71-80$ & - & 2 & 1 & - & - & - & - & - & - & 2 & - & - & 5 & 2,87 \\
\hline $81-90$ & - & 2 & - & - & - & - & - & - & 1 & 1 & - & - & 4 & 2,29 \\
\hline $91-100$ & - & - & - & - & 1 & - & - & - & - & - & - & - & 1 & 0,57 \\
\hline Total & 8 & 31 & 14 & 6 & 12 & 14 & 15 & 14 & 11 & 29 & 9 & 11 & 174 & 100 \\
\hline \multicolumn{15}{|l|}{ Escolaridade } \\
\hline Ens. Fund. & & & & & & & & & & & & & & \\
\hline $\begin{array}{l}\text { Completo } \\
1^{\mathrm{a}} \text { a } 4^{\mathrm{a}} \text { série }\end{array}$ & - & - & - & - & - & 2 & 1 & 2 & - & 2 & 1 & 1 & 9 & 5,17 \\
\hline incompleta & 1 & 1 & 3 & - & 3 & 4 & 4 & 2 & 6 & 8 & - & 4 & 36 & 20,6 \\
\hline incompleta & 4 & 7 & 1 & - & 3 & 2 & 1 & 1 & - & 6 & 3 & 2 & 30 & 17,2 \\
\hline $\begin{array}{l}4^{\mathrm{a}} \text { série } \\
\text { completa }\end{array}$ & - & & - & - & - & - & 2 & 2 & - & 1 & - & & & 517 \\
\hline Ensino médio & & & & & & & & & & & & & & \\
\hline $\begin{array}{l}\text { completo } \\
\text { Ensino médio }\end{array}$ & - & - & - & - & - & - & 1 & - & - & - & 1 & - & 2 & 1,14 \\
\hline incompleto & 1 & - & - & 1 & - & 1 & - & 1 & 1 & 2 & - & 1 & 8 & 4,59 \\
\hline $\begin{array}{l}\text { Educação } \\
\text { superior }\end{array}$ & & & & & & & & & & & & & & \\
\hline completo & - & - & - & 1 & - & - & - & - & - & - & - & - & 1 & 0,57 \\
\hline $\begin{array}{l}\text { Ensino } \\
\text { superior }\end{array}$ & & & & & & & & & & & & & & \\
\hline incompleto & - & - & - & - & - & - & - & - & 1 & - & - & - & 1 & 0,57 \\
\hline Ignorado & - & - & 1 & - & 2 & 1 & - & - & - & 3 & 2 & - & 9 & 5,17 \\
\hline Não se aplica & 2 & - & 2 & 3 & - & 3 & 4 & 1 & 3 & 1 & 1 & - & 20 & 11,4 \\
\hline Em branco & - & 20 & 7 & 1 & 4 & 1 & 2 & 5 & - & 6 & 1 & 2 & 49 & 28,1 \\
\hline Total & 8 & 31 & 14 & 6 & 12 & 14 & 15 & 14 & 11 & 29 & 9 & 11 & 174 & 100 \\
\hline \multicolumn{15}{|l|}{$\begin{array}{l}\text { Zona de } \\
\text { domicilio }\end{array}$} \\
\hline Rural & 4 & 28 & 11 & 4 & 6 & 9 & 5 & 11 & 10 & 16 & 6 & 7 & 117 & 67,2 \\
\hline Urbana & 3 & 1 & 3 & 2 & 3 & 5 & 9 & 3 & 1 & 12 & 3 & 4 & 49 & 28,1 \\
\hline Peri urbana & - & - & - & - & 2 & - & 1 & - & - & - & - & - & 3 & 1,72 \\
\hline Ignorado & 1 & 2 & - & - & 1 & - & - & - & - & - & - & - & 5 & 2,87 \\
\hline Total & 8 & 31 & 14 & 6 & 12 & 14 & 15 & 14 & 11 & 29 & 9 & 11 & 174 & 100 \\
\hline
\end{tabular}


Tabela 2. Distribuição das variáveis epidemiológicas sobre situação de encerramento e variáveis clínicas dos casos de TB notificados no Estado do Paraná entre 2001 a 2012.

\begin{tabular}{|c|c|c|c|c|c|c|c|c|c|c|c|c|c|c|}
\hline \multirow{2}{*}{$\begin{array}{c}\text { Variáveis } \\
\text { Clínicas e } \\
\text { Encerramento }\end{array}$} & \multicolumn{13}{|c|}{$n^{\circ}$} & \multirow[b]{2}{*}{$\%$} \\
\hline & 2001 & 2002 & 2003 & 2004 & 2005 & 2006 & 2007 & 2008 & 2009 & 2010 & 2011 & 2012 & Total & \\
\hline \multicolumn{15}{|l|}{ Forma clinica } \\
\hline $\begin{array}{l}\text { TB Pulmonar } \\
\text { TB }\end{array}$ & 7 & 29 & 11 & 6 & 9 & 12 & 14 & 10 & 11 & 22 & 9 & 10 & 150 & 86,2 \\
\hline & 1 & 2 & 3 & - & 2 & 2 & 1 & 4 & - & 6 & - & 1 & 22 & 12,64 \\
\hline Extrapulmonar & - & 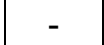 & - & - & 1 & - & - & - & - & 1 & - & 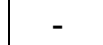 & 2 & 1,14 \\
\hline Total & 8 & 31 & 14 & 6 & 12 & 14 & 15 & 14 & 11 & 29 & 9 & 11 & 174 & 100 \\
\hline \multicolumn{15}{|l|}{$\begin{array}{l}\text { Tratamento } \\
\text { Diretamente } \\
\text { Observado }\end{array}$} \\
\hline Sim & 3 & 14 & - & 2 & 3 & 6 & 8 & 10 & 8 & 24 & 7 & 9 & 94 & 54 \\
\hline Não & 1 & 13 & 7 & 2 & 3 & 5 & 6 & 3 & 3 & 5 & 2 & 2 & 52 & 29,8 \\
\hline Ignorado & - & - & - & - & 1 & - & 1 & 1 & - & - & - & - & 3 & 1,72 \\
\hline Não Refere & 4 & 4 & 7 & 2 & 5 & 3 & - & - & - & - & - & - & 25 & 14,3 \\
\hline Total & 8 & 31 & 14 & 6 & 12 & 14 & 15 & 14 & 11 & 29 & 9 & 11 & 174 & 100 \\
\hline \multicolumn{15}{|l|}{$\begin{array}{l}\text { Situação de } \\
\text { Encerramento }\end{array}$} \\
\hline Cura & 8 & 19 & 8 & 6 & 9 & 8 & 11 & 10 & 8 & 23 & 7 & 9 & 126 & 72,4 \\
\hline Abandono & - & 2 & - & - & - & - & - & 1 & 2 & 4 & 1 & 1 & 11 & 6,32 \\
\hline Óbito por TB & - & - & - & - & - & - & 2 & - & 1 & - & - & - & 3 & 1,72 \\
\hline $\begin{array}{l}\text { multirresistente } \\
\text { Óbito por }\end{array}$ & - & - & - & - & - & - & - & - & - & - & 1 & - & 1 & 0,57 \\
\hline $\begin{array}{l}\text { outras causas } \\
\text { Mudanca de }\end{array}$ & - & - & 2 & - & - & 5 & - & - & - & 1 & - & - & 8 & 4,59 \\
\hline diagnostico & - & 10 & 1 & - & 1 & - & - & 1 & - & - & - & 1 & 14 & 8,05 \\
\hline Transferência & - & - & 3 & - & 2 & 1 & 2 & 2 & - & 1 & - & - & 11 & 6,32 \\
\hline Total & 8 & 31 & 14 & 6 & 12 & 14 & 15 & 14 & 11 & 29 & 9 & 11 & 174 & 100 \\
\hline
\end{tabular}

Tabela 3. Coeficiente de incidência de tuberculose em indígenas no Estado do Paraná no período de 2001 a 2012.

\begin{tabular}{c|c|c|c}
\hline Ano & População & $\mathbf{N}^{\circ}$ de Notificados & $\begin{array}{c}\text { Coeficiente de incidência da TB por } \\
\mathbf{1 0 0} \text { mil habitantes }\end{array}$ \\
\hline 2001 & 23000 & 8 & 34,8 \\
2002 & 10000 & 32 & 320,0 \\
2003 & 15000 & 13 & 86,7 \\
2004 & 11000 & 6 & 54,5 \\
2005 & 21000 & 12 & 57,1 \\
2006 & 29000 & 14 & 48,3 \\
2007 & 27000 & 15 & 55,6 \\
2008 & 38000 & 14 & 36,8 \\
2009 & 33000 & 11 & 33,3 \\
2010 & 26000 & 29 & 111,5 \\
2011 & 18000 & 9 & 50,0 \\
2012 & 16000 & 11 & 68,8 \\
\hline
\end{tabular}




\section{Gráfico 1. Coeficiente de incidência versus percentual de cura entre indígenas no estado do Paraná no período 2001 a 2012.}

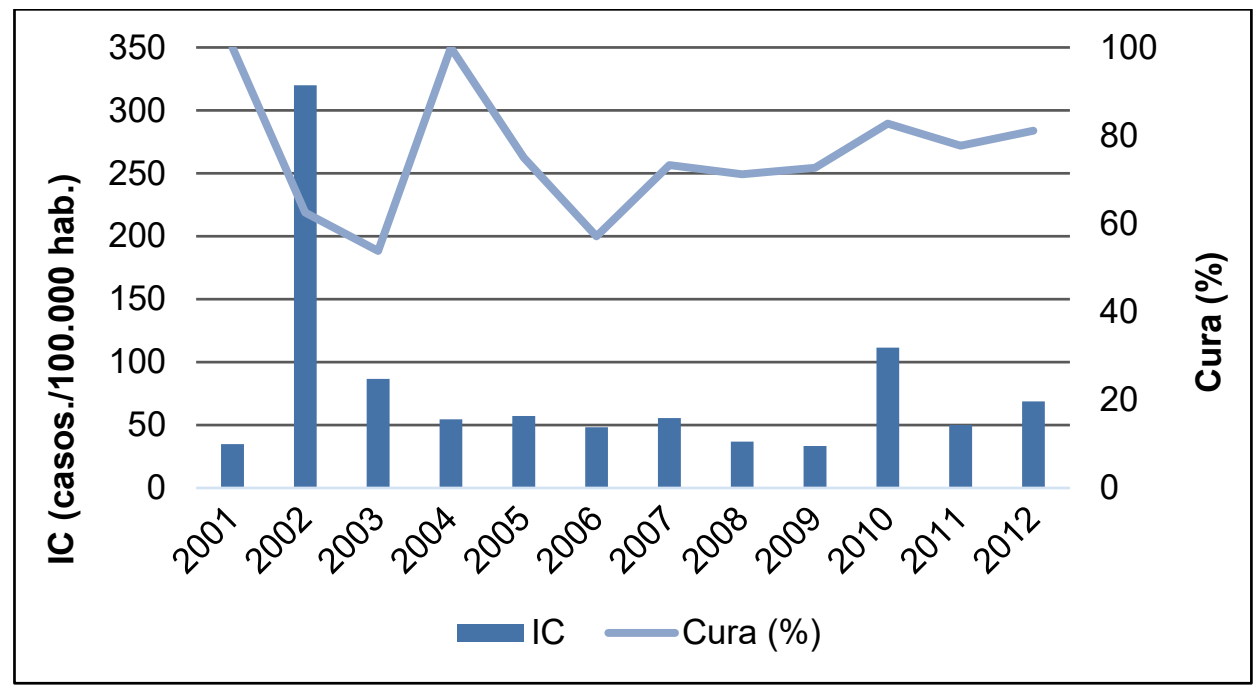

Seguindo tendência de maior escolaridade da população na Região Sul, em relação à Região Norte, verificou-se que os indígenas com TB no Paraná apresentavam mais anos de estudo, do que aqueles da Amazônia onde foi evidenciado maior concentração de casos em pessoas sem nenhum nível de instrução ${ }^{14,19}$.

No que se refere à forma clínica, a presente pesquisa mostrou predominância da TB pulmonar. Destaca-se que este padrão tem sido encontrado em todas as populações estudadas, independentemente, de diferenças populacionais ou étnicas, sendo ela a forma que é responsável pela manutenção da cadeia de transmissão da doença ${ }^{14,20-22}$.

O TDO tem como objetivo principal diminuir as taxas de abandono, e é uma estratégia que está sendo indicada para todas as pessoas identificadas com TB, especialmente para a população vulnerável, abrangendo os povos indígenas ${ }^{12}$.

Contudo, não se registrou alta frequência de tratamento entre indígenas sob o regime do TDO no Paraná, somente em $54 \%$ dos casos, próximo do encontrado no Amazonas e bastante aquém daquele encontrado numa pesquisa realizada no Mato Grosso onde o TDO ocorreu em $88,1 \%$ das vezes ${ }^{20-22}$.
Tendo como base a situação de encerramento, observa-se o percentual de cura de $72,4 \%$, desfecho inferior aos valores fixados (pelo menos $85 \%$ dos casos) pela Organização Mundial da Saúde (OMS) e ao desfecho encontrado entre indígenas com TB com alto percentual de tratamento com TDO ${ }^{12,21}$.

É importante ainda destacar no presente estudo, o abandono do tratamento ficou acima da meta recomendada, máximo $5 \%$ dos casos.

Quando analisado o $\mathrm{Cl}$ da TB em indígenas no Paraná, a série histórica mostrou uma grande variabilidade, devido à circulação do bacilo que pode ser relacionada com condições econômicas desfavoráveis, o acesso limitado aos serviços de saúde, bem como por características das famílias indígenas. Geralmente, as casas indígenas possuem pouca luz natural e ventilação, são pequenas e com grande número de pessoas vivendo no mesmo domicílio favorecendo a disseminação da doença ${ }^{16}$. Ainda, sugere-se fortemente que a persistência de elevados $\mathrm{Cl}$, aponta para a incapacidade do sistema de saúde ${ }^{23}$, em promover o diagnóstico e o tratamento oportuno ${ }^{24}$.

A curva assimétrica na cura mostra a dificuldade operacional em promover o tratamento, devido às questões ligadas ao doente, serviço 
de saúde e fatores ligados à história natural da doença.

As informações desta pesquisa iniciam as discussões sobre a TB entre indígenas no Paraná, outros estudos serão relevantes para melhoria do diagnóstico e tratamento da doença. Verifica-se a necessidade de programar políticas e ações assistências, para alcançar os resultados desejáveis de detecção oportuna e cura da TB na população indígena do estado do Paraná.

Como limitação aponta-se que embora os sistemas de informação em saúde brasileiros tenham alcançado relativas qualidade e confiabilidade com a implementação de medidas de monitoramento e controle, estes ainda são sujeitos a vieses, como o de informação. Pede-se cautela na reprodução dos dados para outros contextos,

\section{REFERÊNCIAS}

1. World Health Organization. Global Tuberculosis Report 2016. Genebra, 2016. Disponível em/Available in: http://apps.who.int/medicinedocs/documents/s23098en/ s23098en.pdf. Acesso em/ Access in: 21 jun 2017.

2. World Health Organization. Global Tuberculosis Report 2012. Genebra, 2012. Disponível em/Available in: http:// www.who.int/tb/publications/global_report/gtbr12_main. pdf. Acesso em/ Access in: 21 jun 2017.

3. Porto OM, Rocha ALC, Branco CAC, Procópio MJ, Hijjar MA. Determinantes sociais, econômicos e culturais das doenças. In: Controle da Tuberculose: uma proposta de integração ensino-serviço. Fundação Oswaldo Cruz. Escola Nacional de Saúde Pública Sergio Arouca. Educação a Distância; coordenado por Maria José Procópio. Rio de Janeiro:2008.

4. Brasil. Ministério da Saúde. Secretaria de Vigilância em Saúde. Boletim Epidemiológico. Indicadores prioritários para o monitoramento do Plano Nacional pelo Fim da Tuberculose como Problema de Saúde Pública no Brasil. 2017;48(8):1-11.

5. Brasil. Ministério da Saúde. Secretaria de Vigilância em Saúde. Boletim Epidemiológico. Tuberculose no Brasil: realidade e perspectivas. 2012; 43(1):15-9

6. Brasil. Ministério da Saúde. Secretaria de Vigilância em Saúde. Boletim Epidemiológico. O controle da tuberculose na população indígena. Brasília: 2013; 44(13):1-9.-

7. Instituto Brasileiro de Geografia e Estatística. Censo Demográfico 2010. Rio de Janeiro: IBGE; 2010. Disponível em/Available in: https://biblioteca.ibge.gov.br/visualizacao/ periodicos/95/cd_2010_indigenas_universo.pdf. Acesso em/ Access in: 20 jun 2017.

8. Instituto Brasileiro de Geografia e Estatística. Indígenas. Rio de Janeiro: IBGE; 2010. Disponível em/Available in: https://indigenas.ibge.gov.br/graficos-e-tabelas-2.html Acesso em/ Access in: 08 fev 2018. visto que estes são oriundos de pessoas que se autodeclararam indígenas durante a notificação do caso.

\section{CONCLUSÃO}

As características dos indígenas com TB no Paraná não se diferenciaram da população não indígena doente por TB. O estudo mostra uma significativa vulnerabilidade dos indígenas, o que torna relevante um esforço adicional frente à assistência prestada à saúde dessa população.

Desta forma, acredita-se que o estudo torna-se relevante e imprescindível para subsidiar, bem como, avaliar as ações de controle da TB em indígenas colaborando para uma diminuição da incidência da doença nesta população.

9. FUNAI (Fundação Nacional do Índio). Terras Indígenas Brasilia: 2017. Disponível em/Available in: http://www. funai.gov.br/index.php/indios-no-brasil/terras-indigenas. Acesso em/ Access in: 23 jun 2017.

10. Brasil. Boletim epidemiológico, ministério da saúde. Tuberculose, população indígena e determinantes sociais. 2014; 45(18):1-13.

11. Santos SC, Marques AMC, Oliveira RL, Cunha RV. Diagnóstico da tuberculose em indígenas menores de quinze anos por meio de um sistema de pontuação em Mato Grosso do Sul. J Bras Pneumol. 2013; 9(1):84-91.

12. Brasil. Ministério da Saúde. Secretaria de Vigilância em Saúde. Departamento de Vigilância Epidemiológica. Manual de recomendações para o controle da tuberculose no Brasil. Brasília: Ministério da Saúde, 2011. Disponível em/ Available in: http://bvsms.saude.gov.br/bvs/publicacoes/ manual_recomendacoes_controle_tuberculose_brasil. pdf. Acesso em/ Access in: 25 jun 2017.

13. Rlos, DPG, Malacarne J, Alves LCC, Santanna CC, Camacho LAB, Basta PC. Tuberculose em indígenas da Amazônia brasileira: estudo epidemiológico na região do Alto Rio Negro. Rev Panam Salud Publica. 2013; 33(1):229.

14. Mendes APM, BastosJL, Bresan D, Leite MS. Situação epidemiológica da tuberculose no Rio Grande do Sul: uma análise com base nos dados do Sinan entre $2003 \mathrm{e}$ 2012 com foco nos povos indígenas. Rev Bras Epidemiol. 2016;19(3):658-69.

15. Escobar AL, Junior CEAC, Camacho LA, Portella MC Tuberculose em populações indígenas de Rondônia, Amazônia, Brasil. Cad Saúde Pública. Rio de Janeiro:2001; 17(2):285-98.

16. Basta PC, Coimbra CE Jr, Welch JR, Correa LCA, Santos RV, Camacho LAB. Tuberculosis among the xavante Indians of the Brazilian Amazon: an epidemiological and ethnographic assessment. Ann Hum Biol. 2010; 37 (5): 643-57. 
17. Bierrenbach AL, Gomes ABF, Noronha EF, Souza MFM. Incidência de tuberculose e taxa de cura, Brasil, 2000 a 2004. Rev Saude Publica. 2007;41(1):24-33.

18. Levino A, Oliveira RM. Tuberculose na população indígena de São Gabriel da Cachoeira, Amazonas, Brasil. Cad Saúde Pública. 2007;23 (7): 1728-1732.

19. Melo TEMP, Resendes APC, Souza-santos R, Basta PC. Distribuição espacial e temporal da tuberculose em indígenas e não indígenas de Rondônia, Amazônia Ocidental, Brasil. Cad Saúde Pública. Rio de Janeiro,2012; 28 (2): 267-80.

20. Belo EN, Orellana JDY,Levino A, Basta PC. Tuberculose nos municípios amazonenses da fronteira Brasil-Colômbia-Peru-Venezuela: situação epidemiológica e fatores associados ao abandono. Rev Panam Salud Publica. Washington, 2013; 34(5):321-9.

21. Orellana JD, Goncalves MJF, Basta PC. Características sociodemográficas e indicadores operacionais de controle da tuberculose entre indígenas e não indígenas de Rondônia, Amazônia Ocidental, Brasil. Rev Bras Epidemiol. São Paulo, 2012;15(4):714-24.
22. Basta PC, Marques M, Oliveira RL, Cunha EAT, Resendes APC, Souza-Santos R.Social inequalitiesandtuberculosis: ananalysisbyrace/color in Mato Grosso do Sul, Brazil. Rev Saude Publica. 2013; 47(5):854-64.

23. Souza MSPL, Aquino R, Pereira SM, Pereira SM, Costa $\mathrm{MCN}$, Barreto ML et al. Fatores associados ao acesso geográfico ao serviço de saúde por pessoas com tuberculose em três capitais do Nordeste brasileiro. Cad Saúde Pública.2015; 31(1):111-20.

24. Viana PVS, Gonçalves MJF, Basta PC.Ethnic and racial inequalities in notified cases of tuberculosis in Brazil. PLos ONE.2016; 11(5): e0154658

\section{CORRESPONDÊNCIA}

Reinaldo Antonio Silva-Sobrinho

Universidade do Oeste do Paraná - UIOESTE

Av. Tarquínio Joslin dos Santos, 1300

Foz do Iguaçu - PR - Brasil

CEP: 85870-650

E-mail: reisobrinho@unioste.br 